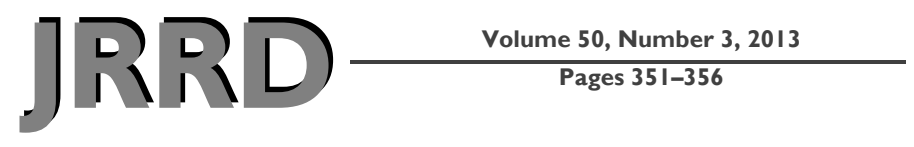

\title{
Sex, shoulder pain, and range of motion in manual wheelchair users
}

\author{
Karla K. Wessels, MS, ATC; ${ }^{1 *}$ Jennifer L. Brown, MS, ATC; ${ }^{2}$ Kyle T. Ebersole, PhD, ATC; ${ }^{3}$ Jacob J. Sosnoff, PhD $^{1}$ \\ ${ }^{1}$ Department of Kinesiology and Community Health, University of Illinois at Urbana-Champaign, Urbana, IL; ${ }^{2}$ Campus \\ Recreation and Intramurals, Georgia Southern University, Statesboro, GA; ${ }^{3}$ Department of Kinesiology, University of \\ Wisconsin-Milwaukee, Milwaukee, WI
}

\begin{abstract}
Upwards of $70 \%$ of manual wheelchair users (MWUs) experience shoulder pain. Pain is more prevalent among females than males. The causes of this sex discrepancy are not understood. Decreased range of motion (ROM) has been suggested as a major contributor, but the interaction of sex, ROM, and shoulder pain has not been investigated, thus the purpose of this investigation. We divided 30 MWUs (18 males, 12 females; $21.93+/-3.77 \mathrm{yr}$ ) into two groups based on self-reported shoulder pain: pain group ( $n=14 ; 9$ males, 5 females) and no pain group ( $n=16$; 9 males, 7 females). We used a digital goniometer to assess ROM. Participants' shoulder active and passive ROMs were tested bilaterally on the following joint motions: flexion, extension, abduction, adduction, internal rotation, and external rotation. We used a visual analog scale to assess shoulder pain. Of the participants, $47 \%$ reported shoulder pain. Overall, the no pain group had greater ROM than the pain group, with further analysis revealing this association was only significant in females during extension $(p<0.05)$. ROM impairments were only present in extension in females with shoulder pain. The mechanism underlying this sex difference is not clear.
\end{abstract}

Key words: adapted athletics, disability, glenohumeral joint, flexibility, range of motion, sex, shoulder, shoulder pain, visual analog scale, wheelchair.

\section{INTRODUCTION}

In the United States, an estimated 1.5 million individuals use manual wheelchairs [1]. This population is expected to increase because of the combination of the ongoing military conflicts and the effects of advanced medical care on survival rates [1]. It is not surprising that manual wheelchair users (MWUs) experience shoulder pain given the persistent use of their shoulders in activities of daily living. Indeed, upwards of 70 percent of MWUs experience pain [2-7]. An array of factors could contribute to shoulder pain.

One reported observation of shoulder pain and MWUs is a sex bias. In a sample of 80 MWUs (22 female), it was shown that shoulder pain was more prevalent in females [6]. Similarly, in an additional study it was found that shoulder pain is more severe in female MWUs than in males [8]. However, the underlying mechanisms driving this sex discrepancy have not yet been elucidated.

A potential factor contributing to sex differences in shoulder pain in MWUs is shoulder joint range of motion (ROM). It is documented in ambulatory populations that females have greater shoulder joint ROM than their male counterparts [9]. It has been proposed that increased ROM in females places them at greater risk for shoulder injuries [9]. It is logical to speculate that increased ROM in female nondisabled populations will also be found in manual wheelchair populations; however, there is minimal evidence of sex differences in ROM in MWUs [10].

\footnotetext{
Abbreviations: $\mathrm{MWU}=$ manual wheelchair user, $\mathrm{ROM}=$ range of motion, $\mathrm{SCI}=$ spinal cord injury, $\mathrm{T}=$ thoracic, VAS = visual analog scale.

*Address all correspondence to Karla K. Wessels, MS, ATC; University of Illinois, Department of Kinesiology and Community Health, 1207 S Oak St, Champaign, IL 61820; 217-300-2220. Email: karlawessels1@gmail.com http://dx.doi.org/10.1682/JRRD.2011.02.0025
} 
In contrast, shoulder pain has been reported to negatively influence ROM. In male MWUs, an association between shoulder pain and decreased shoulder ROM has been observed [3]. The decreased ROM was commonly due to stiffening of soft tissue, narrowing of the acromioclavicular joint, or heterotopic ossification. However, this investigation [11] did not examine whether this association was mediated by sex.

To better understand the contributing factors causing the sex discrepancy in shoulder pain in MWUs, this study examines the relationship between sex, ROM, and shoulder pain.

We hypothesize that females will have greater shoulder ROM than males and shoulder pain will negatively affect shoulder ROM. A better understanding of the association between ROM and shoulder pain can lead to further research on prevention and treatment protocols for shoulder pain in MWUs and whether or not they should be sex specific.

\section{METHODS}

\section{Subjects}

We recruited 30 individuals (18 males, 12 females) who used ergonomically fitted manual wheelchairs as their primary means of locomotion. All participants were active wheelchair athletes. They ranged in age from 18 to $35 \mathrm{yr}$, with a mean of $21.93 \pm 3.77 \mathrm{yr}$. Reason for wheelchair use included lower-limb amputation (3), spina bifida (5), cerebral palsy (1), spinal cord injury (SCI) thoracic (T)1-T9 (5), SCI T10-lumbar 5 (7), acute transverse myelitis (3), and other (6). Average wheelchair use per day was $13.6 \pm 4.14 \mathrm{~h}$ and average length of wheelchair use was $11.57 \pm 5.89$ yr. Participants were divided into two groups (pain and no pain) based on self-report of shoulder pain. Participants were asked if they were currently experiencing shoulder pain and then completed a visual analog scale (VAS) on current shoulder pain experienced in each shoulder. The pain group consisted of 14 participants (9 males, 5 females) and the no pain group consisted of 16 participants (9 males, 7 females) (Table 1).

\section{Procedures}

Upon arrival at the laboratory, subjects provided informed consent and demographic information and completed pain questionnaires. Self-reported demographic information collected included weight, sex, age, physical
Table 1.

Average demographics of participants.

\begin{tabular}{lcc}
\hline \multicolumn{1}{c}{ Variable } & No Pain Group & Pain Group \\
\hline Age (yr) & $21.69 \pm 4.19$ & $22.21 \pm 3.38$ \\
Weight (lb) & $142.00 \pm 25.96$ & $128.88 \pm 40.35$ \\
Males/Females $(n)$ & $9 / 7$ & $9 / 5$ \\
Length of Wheelchair & $12.25 \pm 6.84$ & $12.00 \pm 4.17$ \\
$\quad$ Use (yr) & & \\
Wheelchair Use/Day (h) & $14.60 \pm 2.80$ & $12.96 \pm 5.33$ \\
\hline \hline
\end{tabular}

activity levels, duration of wheelchair use, and average amount of time in a wheelchair per day (Table 1).

ROM was assessed with a digital goniometer (Tracker Freedom, JTECH Medical Inc; Salt Lake City, Utah). Participants were tested on the following shoulder ROMs: flexion, extension, abduction, adduction, internal rotation, and external rotation. The participants were measured bilaterally while seated in a stationary chair for all of the motions. To reduce any limitation because of level of injury, participants were strapped at the waist and thighs to a chair so full ROM could be assessed without instabilities. All ROMs were assessed actively and passively. During active ROM, the participants were instructed to move the arm to the desired end ROM that was comfortable and did not cause pain. For passive ROM, the arm was moved by an investigator through the desired ROM until a comfortable end point had been reached that did not cause pain to the participant. Each motion was tested three times to properly assess any impairment in ROM [12]. During each measurement, the goniometer was lined up along the long axis of the upper arm [12]. For brevity, the average of left and right shoulder ROMs was used in analysis.

A VAS was given by each participant to quantify shoulder pain. The subject marked a line on the VAS in reference to the amount of pain they were currently experiencing in each shoulder at the beginning of the assessment. The VAS is measured in centimeters with 0 being no pain and 10 being maximum pain. The VAS has been found to be both reliable and valid $[7,13]$.

\section{Statistical Analysis}

The average of the three trials of ROM for each shoulder individual motion was placed in a mixed model three-way analysis of variance with group (pain, no pain) and sex (male, female) as between-subject factors and activation (active, passive) as the within-subject factor. When appropriate, Tukey post hoc analysis was used to decipher significant interactions. Significance was set at 
$p<0.05$. All two- and three-way interactions were examined, and only significant findings are reported. SPSS v.16 (IBM; Armonk, New York) was used for all data analysis.

\section{RESULTS}

Of the participants, 47 percent $(n=14)$ indicated they experienced shoulder pain. As expected, participants assigned to the pain group had significantly higher levels of self-reported shoulder pain than those who did not report shoulder pain $(F(1,27)=18.832, p>0.05$; VAS no pain $=0.87 \pm 0.89$ vs pain $=4.23 \pm 2.66$ ). A chi-squared analysis determined there were no group differences with regards to sex, age, weight, length of wheelchair use, or amount of wheelchair use per day. Within the sample, the most common disability present was SCI, followed by spina bifida.

ROM as a function of sex, pain group, and activation is reported in Table 2. The effects of sex, pain, and activation are detailed subsequently.

\section{Shoulder Pain}

A pain group effect was found on ROM during extension $(F(29)=6.143 ; p<0.05)$. This effect resulted from the pain group experiencing less ROM than the no pain group $\left(45.92^{\circ} \pm 3.55^{\circ}\right.$ vs $\left.57.89^{\circ} \pm 3.27^{\circ}\right)$.

\section{Active/Passive Differences}

Statistical analysis revealed a main effect for activation for flexion, extension, external rotation, and adduction. In these directions, passive ROM was greater than active ROM (flexion: $p<0.05,194.55^{\circ} \pm 2.18^{\circ}$ vs $185.13^{\circ} \pm$ $2.43^{\circ}$; extension: $p<0.05,57.50^{\circ} \pm 3.80^{\circ}$ vs $46.30^{\circ} \pm$ $3.00^{\circ}$; external rotation: $p<0.05,118.95^{\circ} \pm 2.73^{\circ}$ vs $103.04^{\circ} \pm 3.12^{\circ}$; adduction: $p<0.05,58.11^{\circ} \pm 6.04^{\circ}$ vs $\left.41.14^{\circ} \pm 1.21^{\circ}\right)$.

\section{Sex Differences}

Statistical analysis revealed a main effect for sex for flexion, extension, and external rotation ROM. Females had greater ROM than males in all three of these directions (flexion: $194.28^{\circ} \pm 2.86^{\circ}$ vs $185.41^{\circ} \pm 2.24^{\circ}$; extension: $57.50^{\circ} \pm 3.80^{\circ}$ vs $46.30^{\circ} \pm 2.98^{\circ}$; external rotation: $119.05^{\circ} \pm 4.04^{\circ}$ vs $\left.102.93^{\circ} \pm 3.17^{\circ}\right)$.

\section{Sex, Shoulder Pain, and Range of Motion Difference}

Statistical analysis revealed a significant sex by pain by activation interaction during extension $(F(1,29)=$ $6.00 ; p<0.05)$. Post hoc analysis revealed that the interaction between sex and pain group resulted from females with pain experiencing significantly less ROM than females without pain, while there was no difference between males with and without pain (females no pain $69.41^{\circ} \pm 4.91^{\circ}$ vs pain $45.60^{\circ} \pm 5.80^{\circ}$; males no pain $46.37^{\circ} \pm 4.33^{\circ}$ vs pain $46.23^{\circ} \pm 4.10^{\circ}$ ).

\section{DISCUSSION}

The purpose of this investigation was to determine whether there is a sex difference in shoulder ROM in MWUs and whether there is a differential influence of shoulder pain on ROM in male and female MWUs. We hypothesized that females would have greater ROM and that shoulder pain would negatively affect shoulder ROM. Overall, we found that 47 percent of the population

Table 2.

Average range of motion (degrees) as function of sex, pain, and activation.

\begin{tabular}{|c|c|c|c|c|c|c|c|c|}
\hline Sex & Group & Activation & Flexion & Extension & Abduction & Adduction & $\begin{array}{l}\text { External } \\
\text { Rotation }\end{array}$ & $\begin{array}{l}\text { Internal } \\
\text { Rotation }\end{array}$ \\
\hline \multirow[t]{4}{*}{$\overline{\text { Male }}$} & No Pain & Active & $184 \pm 4$ & $43 \pm 3$ & $178 \pm 5$ & $43 \pm 2$ & $94 \pm 6$ & $66 \pm 6$ \\
\hline & & Passive & $190 \pm 4$ & $50 \pm 9$ & $181 \pm 11$ & $54 \pm 11$ & $106 \pm 5$ & $62 \pm 6$ \\
\hline & Pain & Active & $180 \pm 4$ & $37 \pm 3$ & $169 \pm 5$ & $40 \pm 2$ & $96 \pm 5$ & $57 \pm 6$ \\
\hline & & Passive & $189 \pm 4$ & $55 \pm 9$ & $183 \pm 10$ & $52 \pm 10$ & $115 \pm 5$ & $53 \pm 5$ \\
\hline \multirow[t]{4}{*}{ Female } & No Pain & Active & $195 \pm 5$ & $52 \pm 3$ & $193 \pm 6$ & $41 \pm 2$ & $125 \pm 6$ & $67 \pm 7$ \\
\hline & & Passive & $203 \pm 4$ & $87 \pm 11$ & $178 \pm 13$ & $75 \pm 12$ & $133 \pm 6$ & $69 \pm 6$ \\
\hline & Pain & Active & $182 \pm 6$ & $40 \pm 4$ & $184 \pm 7$ & $40 \pm 3$ & $97 \pm 8$ & $76 \pm 9$ \\
\hline & & Passive & $197 \pm 5$ & $51 \pm 13$ & $187 \pm 15$ & $52 \pm 15$ & $121 \pm 7$ & $64 \pm 8$ \\
\hline
\end{tabular}


experienced shoulder pain. Of the participants, 50 percent of the males reported shoulder pain, while 42 percent of the females reported shoulder pain. Females had greater ROM than males in shoulder flexion, extension, and external rotation. There was no effect of shoulder pain on ROM in males. However, females with shoulder pain had reduced ROM and were affected by shoulder pain only in extension.

\section{Shoulder Pain}

In the current investigation, 47 percent of the participants reported shoulder pain, which was within the expected range. This observation is congruent with previous reports, which show a range from 30 to 74 percent [2-7]. The mechanisms underlying shoulder pain in MWUs are multifaceted. For instance, it is maintained that as MWUs age they more commonly report shoulder pain [2-5]. It has also been suggested that wheelchair athletes experience less shoulder pain than nonathletes $[2,5]$. Given that these were young ( 20 yr) and physically active wheelchair users, it is alarming that almost half of the population tested in the current study suffered shoulder pain.

\section{Active/Passive Differences}

Passive ROM was greater than active ROM in flexion, external rotation, adduction, and extension. It was unexpected that passive ROM was not greater than active $\mathrm{ROM}$ in each direction. It is maintained that passive $\mathrm{ROM}$ is greater since during active movement a muscle actively contracts and minimizes the elastic elements (e.g., slack) and causes a resistance to movement. While in passive movement, an external force is applied and the limb is capable of moving farther.

However, passive ROM was not consistently greater than active ROM across all joint motions. For instance, there were no differences between passive and active motion in abduction and internal rotation. It is possible that anatomical limitations equally constrain arm abduction and internal rotation in both passive and active movement.

\section{Sex Difference}

Females had significantly greater ROM than males only in flexion, extension, and external rotation. Most likely, a multitude of interacting factors drive sex differences in ROM. It is possible muscle mass [9], laxity [9], and activity levels [2,5] affect ROM differentially across joint motions. Previous studies have shown that nondisabled females have greater ROM than males throughout the shoulder [9]; however, females had greater ROM in only half of the joint motions tested here. One possibility for the sex-directional difference in MWUs could be sexrelated anatomical differences or differences in muscle mass. For instance, males tend to have more muscle mass in their shoulders [9]. This increased mass could impede movement and consequently result in less ROM than females. Specifically, the sex difference in flexion and extension could be due to males having greater pectoralis muscle group and deltoid mass. In contrast, external rotation differences could result from females having greater laxity [9].

Another possible reason for the lack of a greater sex difference in the current investigation is the use of active participants (i.e., wheelchair athletes). Previous investigations have suggested that physically active females have less laxity than sedentary females [9]. However, in an investigation of physically active and sedentary wheelchair users, no difference in ROM was observed between groups. Note that there was no examination of the effect of sex in the previous study [10]. Further work is needed to clarify the association between sex, shoulder ROM, and activity in wheelchair users.

\section{Sex, Shoulder Pain, and Range of Motion}

The novel finding in the current investigation was that only female MWUs had ROM deficits associated with shoulder pain. We expected pain to negatively affect both sexes; however, this did not hold true. This observation extends and clarifies previous work that reported MWUs with ROM deficits were more likely to report shoulder pain [3]. It is also unique that the females were only affected by pain during extension. The females who experienced shoulder pain during extension had ROM similar to males without shoulder pain. During wheelchair propulsion, the shoulder bears the most loading when extended and internally rotated [14]. In the current investigation, females had more extension than males, so it is possible that with greater ROM the females bear loading at a greater angle, thus exposing the shoulder to more injury.

It cannot be determined within the current data set whether the shoulder pain caused decreased ROM or vice versa. The differential association with shoulder pain and ROM needs to be further examined to determine whether or not there is a causal link. 


\section{Implications}

Females having greater ROM than males could predispose them to injuries. Greater laxity and ROM appear to be contributing factors to increased incidence of anterior cruciate ligament injuries in females [15-16]. Having greater joint laxity could potentially put females at greater risk for injuries. The ROM and injury discrepancy between the sexes suggests that there could be a need for sex-specific treatment and prevention for shoulder pain.

\section{Limitations}

A possible limitation of this study is that the sample tested was composed of collegiate athletes. Their ROM is not representative of the general wheelchair population because of training regimens. Another limitation is the age of the population. The sample was relatively young, and it has been shown that more structural changes occur with age that could be detrimental to ROM [3]. It is possible that with advanced age there could be greater sex and pain effects on ROM. A variety of disabilities were present in this sample. It is possible differences in trunk control and muscle tone could have affected ROM, although we attempted to minimize this effect. Additionally, the sample size was relatively small and could not be representative of the general population.

\section{CONCLUSIONS}

Of the current study population, 47 percent reported shoulder pain. ROM impairments were present only in females with shoulder pain, although the causal link between the two cannot be determined. Passive ROM was greater than active ROM; however, this did not extend through all ROMs. Females had significantly greater ROM than males in flexion, extension, and external rotation. Further research is needed to determine the cause of the differential association between ROM, shoulder pain, and sex. Future research can determine if this is an occurrence in the general MWU population or if it is specific to the active MWUs. Sex differences in skeletomuscular composition could potentially contribute to the differential association between pain and ROM. Sex-specific treatment for shoulder pain in MWUs may be necessary.

\section{ACKNOWLEDGMENTS}

\section{Author Contributions:}

Study concept and design: K. K. Wessels, J. J. Sosnoff, K. T. Ebersole. Acquisition of data: K. K. Wessels, J. L. Brown.

Analysis and interpretation of data: K. K. Wessels, J. J. Sosnoff. Drafting of manuscript: K. K. Wessels, J. J. Sosnoff.

Critical revision of manuscript for important intellectual content:

J. J. Sonoff, K. T. Ebersole.

Statistical analysis: K. K. Wessels, J. J. Sosnoff.

Study supervision: J. J. Sosnoff.

Financial Disclosures: The authors have declared that no competing interests exist.

Funding/Support: This material was not funded.

Institutional Review: This study was approved by the institutional review board at the University of Illinois-Urbana, Champaign.

Participants provided informed consent.

Participant Follow-Up: The authors have no plans to notify the study subjects of the publication of this article because of lack of contact information.

\section{REFERENCES}

1. Kaye HS, Kang T, LaPlante MP. Mobility device use in the United States. Disability statistics report 14 . Washington (DC): NIDDR; 2000.

2. Alm M, Saraste H, Norrbrink C. Shoulder pain in persons with thoracic spinal cord injury: prevalence and characteristics. J Rehabil Med. 2008;40(4):277-83. [PMID:18382823] http://dx.doi.org/10.2340/16501977-0173

3. Ballinger DA, Rintala DH, Hart KA. The relation of shoulder pain and range-of-motion problems to functional limitations, disability, and perceived health of men with spinal cord injury: a multifaceted longitudinal study. Arch Phys Med Rehabil. 2000;81(12):1575-81. [PMID:11128892] http://dx.doi.org/10.1053/apmr.2000.18216

4. Curtis KA, Drysdale GA, Lanza RD, Kolber M, Vitolo RS, West R. Shoulder pain in wheelchair users with tetraplegia and paraplegia. Arch Phys Med Rehabil. 1999;80(4):453-57. [PMID:10206610] http://dx.doi.org/10.1016/S0003-9993(99)90285-X

5. Fullerton HD, Borckardt JJ, Alfano AP. Shoulder pain: a comparison of wheelchair athletes and nonathletic wheelchair users. Med Sci Sports Exerc. 2003;35(12):1958-61. [PMID:14652488] http://dx.doi.org/10.1249/01.MSS.0000099082.54522.55

6. Gutierrez DD, Thompson L, Kemp B, Mulroy SJ; Physical Therapy Clinical Research Network; Rehabilitation Research and Training Center on Aging-Related Changes in Impairment for Persons Living with Physical Disabilities. The relationship of shoulder pain intensity to quality of life, physical activity, and community participation in 
persons with paraplegia. J Spinal Cord Med. 2007;30(3): 251-55. [PMID:17684891]

7. Jensen MP, Hoffman AJ, Cardenas DD. Chronic pain in individuals with spinal cord injury: a survey and longitudinal study. Spinal Cord. 2005;43(12):704-12.

[PMID:15968299]

http://dx.doi.org/10.1038/sj.sc.3101777

8. Sosnoff JJ, Wessels KK, Brown JL, Ebersole KT. Shoulder pain and gender in manual wheelchair users. Med Sci Sports Exerc. 2009;41:506.

http://dx.doi.org/10.1249/01.MSS.0000356093.53609.3d

9. Borsa PA, Sauers EL, Herling DE. Patterns of glenohumeral joint laxity and stiffness in healthy men and women. Med Sci Sports Exerc. 2000;32(10):1685-90.

[PMID:11039638]

http://dx.doi.org/10.1097/00005768-200010000-00004

10. Finley MA, Rodgers MM. Prevalence and identification of shoulder pathology in athletic and nonathletic wheelchair users with shoulder pain: A pilot study. J Rehabil Res Dev. 2004;41(3B):395-402. [PMID:15543457] http://dx.doi.org/10.1682/JRRD.2003.02.0022

11. Bekkering WP, ten Cate R, van Suijlekom-Smit LW, Mul D, van der Velde EA, van den Ende CH. The relationship between impairments in joint function and disabilities in independent function in children with systemic juvenile idiopathic arthritis. J Rheumatol. 2001;28(5):1099-1105. [PMID:11361196]

12. Gunnar BS, Cocchiarella L. (2000). The Guides to the evaluation of permanent impairment. 5th ed. New York (NY): American Medical Association Press; 2000.

13. Good M, Stiller C, Zauszniewski JA, Anderson GC, Stanton-Hicks M, Grass JA. Sensation and Distress of Pain
Scales: reliability, validity, and sensitivity. J Nurs Meas. 2001;9(3):219-38. [PMID:11881266]

14. Collinger JL, Boninger ML, Koontz AM, Price R, Sisto SA, Tolerico ML, Cooper RA. Shoulder biomechanics during the push phase of wheelchair propulsion: a multisite study of persons with paraplegia. Arch Phys Med Rehabil. 2008;89(4):667-76. [PMID:18373997] http://dx.doi.org/10.1016/j.apmr.2007.09.052

15. Quatman CE, Ford KR, Myer GD, Paterno MV, Hewett TE. The effects of gender and pubertal status on generalized joint laxity in young athletes. J Sci Med Sport. 2008;11(3): 257-63. [PMID:17597005] http://dx.doi.org/10.1016/j.jsams.2007.05.005

16. Adachi N, Nawata K, Maeta M, Kurozawa Y. Relationship of the menstrual cycle phase to anterior cruciate ligament injuries in teenaged female athletes. Arch Orthop Trauma Surg. 2008;128(5):473-78. [PMID:17909824] http://dx.doi.org/10.1007/s00402-007-0461-1

Submitted for publication February 8, 2012. Accepted in revised form July 13, 2012.

This article and any supplementary material should be cited as follows:

Wessels KK, Brown JL, Ebersole KT, Sosnoff JJ. Sex, shoulder pain, and range of motion in manual wheelchair users. J Rehabil Res Dev. 2013;50(3):351-56.

http://dx.doi.org/10.1682/JRRD.2011.02.0025

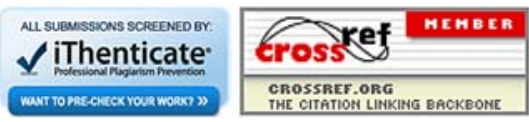

